\title{
Negotiation Protocol for Agile and Reliable E-Science Collaboration
}

\author{
Zeqian Meng \\ School of Computer Science \\ The University of Manchester \\ 1.17, Kilburn Building, Oxford Road \\ Manchester, M13 9PL \\ Email: zeqian.meng@manchester.ac.uk
}

\author{
John Brooke \\ School of Computer Science \\ The University of Manchester \\ J1, Sackville Street Building \\ Sackville Street, Manchester, M1 3BB \\ Email: John.Brooke@manchester.ac.uk
}

\section{THE PROTOCOL}

Our protocol is based on [1], which is designed upon Alliance model and European Union (EUs) Electronic Commerce Directive, leading law-based negotiation between resource requesting organisation and resource providing organisation. Many of the messages identified in [1] correspond to negotiation messages in this paper. The key differences between the protocol described in this paper to [1] include: the introduction of Resource Manager, and support for access negotiation for collaboration formation; negotiation results notification from provider to manager to manage complete collaboration lifecycle; and termination negotiation considers termination request from Resource Manager. Furthermore, race conditions, which are not addressed in [1], will be discussed in this protocol.

As to terminate a contract before normal completion in a business scenario may allow the innocent party to claim monetary penalty, we distinguish different final states for negotiation procedure and termination procedure. A resource negotiation process terminates in one of three possible agreed outcomes: contracted, non-contracted, terminated; while termination negotiation ends with provider terminated, requester terminated, and coterminated.

\section{A. Terminology}

There are three participants defined in our negotiation protocol: Resource Requester, Resource Manager, and Resource Provider. Resource Requester can be a scientist, who wants to take advantage of computing facility to explore and analyse his/her research, and may have limited IT-related skills. Resource Manager coordinates resource sharing among users from different administrative organisations, by controlling user's access to shared resources. Resource Provider is the entity, which can attain monetary rewards by allowing external users to access local resources, via reaching an agreement for resource usage with Requester collaboration and subsequently users, while it still can keep autonomy of its resources. Resources that can be shared include computational resources, storage, data, and network, etc. The lifecycle of collaborative resource sharing does not only consider about negotiation for resource usage, but also the access control between Resource Requester organisation and Resource Provider.

Negotiation is a way to resolve difference and reach an agreement among parties, usually with multiple rounds of communication to reach an agreement. Re-negotiation is the procedure that a party of a contracted agreement wants to change terms in that existing contract. This will terminate previous contract and activate new negotiation for possible agreement.

\section{B. Assumptions and Rules}

The following identifies the boundaries of the designed protocol:

1) It defines participants, their messaging behaviours, and message types.

2) It includes a negotiation protocol and a contract termination protocol.

3) It identifies race conditions that each participant may encounter during negotiation respectively, with corresponding agreed outcomes.

4) Notification messages during service execution will not be discussed. For example, messages to inform Requester that the specified input file has been transferred or deleted. Actually, these features can be provided by existing infrastructures.

5) The protocol does not include mechanisms to deal with concurrent communication, where a party has to deal with multiple messages from different sources at the same time. However, related work can be considered for future testbed implementation. HARC (The HighlyAvailable Resource Co-allocation) [2] is one of such examples. It is proposed for receiving multiple resource supply in a coordinated and resilient manner, by replacing a single coordinator in classic two-phase commit (2PC) protocol with multiple coordinators.

6) There is no mechanism to deal with multi-peer consensuses, where a group of users want to reach an agreement.

7) The negotiation protocol so far concentrates on negotiation procedure and resource/service description languages. It does not consider law-related contract con- 
tents so far. However, experience for future work can be learned from proposed contract templates for scientific Grid/Cloud collaboration, including commercial Clouds. For example, the FitSM templates used by EGI pay-byuse experiment [3], which 'is a lightweight standards, aiming at facilitating service management in IT service provision, including federated scenarios' [4].

\section{Protocol Specification}

The protocol messages are grouped by different phases for negotiation: pre-negotiation, negotiation, and termination, while the negotiation procedure is sub-sectioned according to functionalities: resource negotiation, access negotiation, and revocation.

To avoid denial of service for providers and make it compatible with existing e-Science collaboration models, an Offer message can only be sent from requester to provider, and the decision to accept or reject an Offer is made by provider in this protocol. However, Quote and QuoteRequest are designed for both parties to express the intention for collaboration, which are not legally bound to final contracts. If requester wants to change contract contents before forming an agreement, revocation may be activated by sending a revoke request. Access negotiation allows Resource Manager controls resource allocation, while termination negotiation enables three parties to terminate a contract before normal completion.

1) Pre-negotiation: QuoteRequest: message from Resource Requester to Resource Provider that asks for a nonbinding estimate of available resources.

Quote: message from Resource Provider to Resource Requester to inform available resources for possible collaboration. A Quote is different from Offer and cannot be accepted, indicating an invitation for Offers without legal effects.

2) Resource Negotiation: Offer: message from Resource Requester to Resource Provider that initiates a contract negotiation. It may specify the resource details and performance requirements.

OfferAck: message from Resource Provider to Resource Requester that informs the arrival of an Offer, before making decisions. This message aims at support non-blocking communication on Resource Requester's program, which is especially true on systems where communication can be executed autonomously.

Accept: message from the provider to inform the Resource Requester that an Offer has been accepted, which can only happen after requester's access privileges have been approved by Resource Manager.

AcceptAck: message from Resource Requester to Resource Provider to commute the arrival of Accept message. This is essential in a commercial scenario, which requires that the acceptance must be communicated to offeree.

Reject: message from Resource Provider to Resource Requester, indicating required resource or performance cannot be satisfied. This can only happen after requester's access privileges have been approved by Resource Manager. However, it does not indicate the end of a negotiation, where Resource
Requester can send a new Offer to continue negotiation. The Reject message may contain reasons for rejection, contributing to higher possibility to research an agreement in following communication.

Terminate: all three parties can send a Terminate any time during negotiation, which ends the conversation. As it happens before a contract being formed and no responsibility bound yet, there is no need to distinguish which party terminates the negotiation. However, it is necessary to ensure that the three parties are in the same consistent state: if the Terminate is sent from Resource Manager to Resource Provider to end the negotiation between Resource Provider and Resource Requester, a Terminate message should be sent from provider to requester, indicating that the termination is required by Resource Manager; when the Terminate is sent either from requester to provider, or from provider to requester, the provider should inform Resource Manager that the negotiation fails.

Contracted: message from Resource Provider to Resource Manager to inform successful contract formation, which enables Resource Manager to update access control and resource usage information. It closes the negotiation.

Uncontracted: message from Resource Provider to $\mathrm{Re}$ source Manager to indicate a failed negotiation, corresponding to an access negotiation before. This leads to an end state of negotiation.

3) Access Negotiation: AccessReq: message from Resource Provider to Resource Manager for requester's access verification. As discussed by [5], role-based resource management is a method to simply the procedure, by reducing the mapping complexity between users' identities and provider's local access management.

Accessing: message from Resource Provider to Resource Requester to inform access verification is being processed with Resource Manager.

AccessDenied: two paths for an AccessDenied message: AccessDenied from Resource Manager to Resource Provider to indicate denial of requester's request. Denial reasons may be indicated, for example, no privilege or over-expenditure; from Resource Provider to resource requester with reason of denial, indicating Resource Manager rejects requester's request. AccessDenied leads to an end state of negotiation.

AccessSucceed: two paths for an AccessSucceed message: message from Resource Manager to Resource Provider to confirm requester's access; message from Resource Provider to Resource Requester, to inform that requester's access request has been verified by Resource Manager.

4) Requester Revocation: RevokeReq: message from Resource Requester to Resource Provider, after sending an Offer or an Accept, and before sending an AcceptAck. This allows requester to change contract contents before its formation.

RevokeAcc: message from Resource Provider to requester, indicating that the requester's RevokeReq is accepted, and a new Offer can be proposed.

RevokeRej: message from Resource Provider to requester, corresponding to a RevokeReq. After receiving a RevokeRej, 


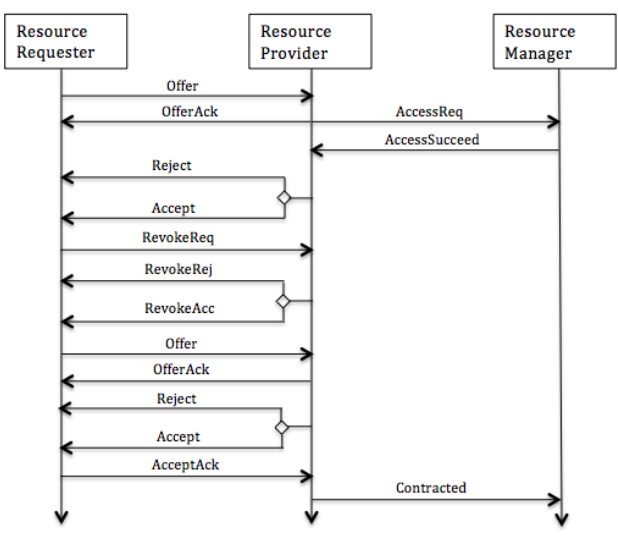

Fig. 1. Negotiation Procedure

requester may choose to send a Terminate to close the negotiation or continue communication.

5) Contract Termination: As mentioned before, renegotiation in this protocol requires destroy of a contract before modifying contents of it. In a business scenario, the termination of a contract before its normal completion may introduce benefit conflicts, making it important to clarify which party terminates an in-effect contract. According to this, this protocol defines additional message types for terminating a contract, while re-negotiation messaging behaviours remain the same as negotiation.

ReqTerminate: message can be sent from either Resource Requester or Resource Manager to Resource Provider, which should indicate which contract is terminated, and may result in penalty as agreed in the terminated contract. If ReqTerminate is sent from Resource Requester, Resource Provider should notify the termination to Resource Manager; while the ReqTerminate is sent from Resource Manager to Resource Provider, Resource Provider should inform Resource Requester with termination.

ProTerminate: message is sent from Resource Provider to both Resource Requester and Resource Manager to terminate a contract, which may include penalty information as agreed in the terminated contract.

6) Race Conditions: To complete the specification, we discuss the messaging situations where requester and provider, or provider and manager send messages that pass by each other on the network while in transit, which is called race condition. For example, requester sends a accept acknowledge before a negotiation time out, but the message is not delivered within the time out period due to message latency. After the negotiation has timed out, provider sends both requester and manager a Terminate message resulting in the two message passing by each other.

As we already consider the cases that Resource Requester/Resource Manager and Resource Provider send Terminate message to terminate a contract at the same time, which results in a CoTerminated state to avoid benefit conflicts, race conditions during a termination negotiation are not discussed here. More details to specify the resulting messaging outcomes for each of the message pairs in resource negotiation and access negotiation are listed below.

Resource Requester Race Conditions Resource Requester may encounter race conditions in situations: after requester sends a message to provider, before receiving the message, provider sends a decision to requester; after requester sends a message to provider, before receiving the message, provider receives Terminate from manager. As requester can send Terminate anytime during negotiation, no matter which message provider sends, after sending Terminate to provider, requester enters un-contracted state.

- If requester sends a RevokeReq, provider sends an OfferAck, an Accessing, an AccessSucceed, or an Accept, requester is in negotiation state and waits for further message from provider, including RevokeRej, RevokeAcc, Terminate. If RevokeRej or RevokeAcc are received, the communication is still in negotiation state; while arrival of Terminate ends negotiation in un-contracted state. Terminate may contain reasons of termination, such as access denied or termination request from Resource Manager.

- If requester sends a RevokeReq, provider sends a Reject, then requester stays in negotiation state, and may send a new Offer to continue negotiation.

- If requester sends a RevokeReq, provider sends an AccessSucceed or Accept, manager sends a Terminate, then negotiation ends in un-contracted state.

- If requester sends a RevokeReq, provider sends a AccessDenied or Terminate, then the negotiation is in uncontracted state.

- If requester sends an AcceptAck, provider or manager sends a Terminate, then the negotiation is in un-contracted state.

Resource Provider Race Conditions Resource Provider may encounter race conditions in situations: after provider sends a message to requester, before receiving the message, requester sends a message to provider; after provider sends a message to requester, before receiving response from requester, provider receives Terminate from manager. As provider can send Terminate anytime during negotiation, no matter which message requester or manager send, after sending Terminate to requester and manager, provider enters un-contracted state.

- If provider sends an OfferAck, an Accessing, an AccessSucceed, or an Accept, requester sends RevokeReq, then provider makes a decision according to the contents in RevokeReq and access decision from Resource Manager if AccessReq has been sent to manager. Decisions include RevokeRej, RevokeAcc, Terminate. If RevokeRej or RevokeAcc are sent, the communication is still in negotiation state; while sending Terminate ends the negotiation in un-contracted state.

- If provider sends a Reject, requester sends a RevokeReq, then provider returns RevokeAcc and stays in negotiation state. 


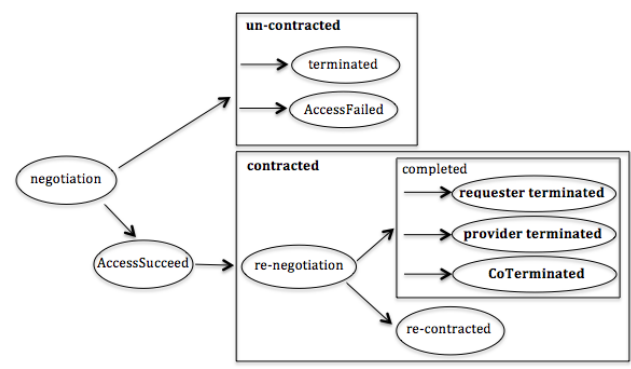

Fig. 2. Negotiation States

- If provider sends an AccessSucceed or Accept, requester sends RevokeReq, manager sends a Terminate, then provider sends requester a Terminate message to inform the termination by manager and negotiation is in an uncontracted state.

- If provider sends a AccessDenied, requester sends RevokeReq or Terminate, then the negotiation ends in uncontracted.

- If provider receives an AcceptAck, manager sends a Terminate, then then a Terminate will be sent to requester indicating termination by Resource Manager and the negotiation ends in un-contracted.

Resource Manager Race Conditions Resource Manager may encounter race conditions in situations: after manager sends a access decision to provider, before receiving the response, provider sends a Terminate to manager; after manager sends a Terminate to provider, before receiving the message, provider sends a negotiation decision to manager;

- If manager sends AccessDenied or AccessSucceed, provider sends a Terminate, then the negotiation ends in un-contracted.

- If manager sends a Terminate, provider sends a Contracted or Uncontracted, then the negotiation ends in uncontracted.

- If manager sends an AccessDenied or AccessSucceed, provider sends an Uncontracted, then the negotiation ends in un-contracted state.

7) Agreed Outcome: The negotiation procedure terminates when Resource Requester, Resource Provider and Resource Manager are in one of their messaging final states: contracted, un-contracted for negotiation procedure, provider terminated, requester terminated, and co-terminated for termination procedure. One important property of the protocol is that the three parties can reach an agreed final outcome at termination. Specifically, two scenarios can lead to a terminated state: one of the three parties sends Terminate to end the conversation during negotiation; either Resource Requester or Resource Manager, and Resource Provider sends Terminate at the same time to terminate a contract.

contracted The negotiation is successful. Resource Requester and Resource Provider reach the contracted state via the AcceptAck messaging state (Resource Requester sends AcceptAck and Resource Provider receives AcceptAck), while
Resource Manager by receiving Contracted message from provider.

un-contracted The negotiation does not reach an agreement. This state is caused by one of the three parties terminates negotiation before reaching an agreement.

provider terminated Resource Provider terminates a contract.

requester terminated Resource Requester or Resource Manager terminates a contract.

coTerminated Resource Provider and Resource Requester/Resource Manager terminate a contract at the same time.

\section{REFERENCES}

[1] Parkin, Michael Stephen. Lightweight client organisations for the computational grid. Diss. University of Manchester, 2007.

[2] MacLaren, Jon. "HARC: the highly-available resource co-allocator." On the move to meaningful internet systems 2007: CoopIS, DOA, ODBASE, GADA, and IS. Springer Berlin Heidelberg, 2007. 1385-1402.

[3] EGI. (2014). EGI Pay-for-Use PoC. Available: https://wiki.egi.eu/wiki/EGI_Pay-for-Use_PoC:Home. Last accessed 05th, January 2015.

[4] Fedsm.eu, (2015). The FitSM Standard - www.fedsm.eu. [online] Available at: http://www.fedsm.eu/ [Accessed 10 Mar. 2015].

[5] Brooke, John M., and Michael S. Parkin. "Enabling scientific collaboration on the Grid." Future Generation Computer Systems 26.3 (2010): 521-530. 